\title{
Coherence manipulation with dephasing-covariant operations
}

\author{
Bartosz Regula $\odot{ }^{1,2, *}$ Varun Narasimhachar, ${ }^{1,2, \dagger}$ Francesco Buscemi, ${ }^{3, \ddagger}$ and Mile Gu ${ }^{1,2,4, \S}$ \\ ${ }^{1}$ School of Physical and Mathematical Sciences, Nanyang Technological University, 637371 Singapore \\ ${ }^{2}$ Complexity Institute, Nanyang Technological University, 637335 Singapore \\ ${ }^{3}$ Graduate School of Informatics, Nagoya University, Chikusa-ku, 464-8601 Nagoya, Japan \\ ${ }^{4}$ Centre for Quantum Technologies, National University of Singapore, 3 Science Drive 2, 117543 Singapore
}

(Received 13 August 2019; published 31 January 2020)

\begin{abstract}
We characterize the operational capabilities of quantum channels which can neither create nor detect quantum coherence vis-à-vis efficiently manipulating coherence as a resource. We study the class of dephasing-covariant operations (DIO), unable to detect the coherence of any input state, as well as introduce an operationally motivated class of channels $\rho$-DIO, which is tailored to a specific input state. We first show that pure-state transformations under DIO are completely governed by majorization, establishing necessary and sufficient conditions for such transformations and adding to the list of operational paradigms where majorization plays a central role. We then show that $\rho$-DIO are strictly more powerful: although they cannot detect the coherence of the input state $\rho$, the operations $\rho$-DIO can distill more coherence than DIO. However, the advantage disappears in the task of coherence dilution as well as generally in the asymptotic limit, where both sets of operations achieve the same rates in all transformations.
\end{abstract}

DOI: 10.1103/PhysRevResearch.2.013109

\section{INTRODUCTION}

Quantum coherence, or superposition, is an intrinsic feature of quantum mechanics that underlies the advantages enabled by quantum information processing and quantum technologies [1]. The resource theory of quantum coherence [1-4] has found extensive use in the characterization of our ability to manipulate coherence efficiently within a rigorous theoretical framework, wherein the properties of a resource are investigated under a suitable set of allowed ("free") operations which reflect the constraints placed on the manipulation of the given resource [5,6]. Despite many promising developments in the establishment of a comprehensive description of coherence, the physical constraints governing its manipulation are unsettled $[1,7,8]$, and one of the most important outstanding questions in the resource theory of quantum coherence is to understand the exact properties of the different operational paradigms under which coherence can be investigated [4,7-26].

Many proposed types of free operations stem from meaningful physical considerations regarding their implementability: for instance, the physically incoherent operations [7] only require the use of incoherent ancillary systems and incoherent

\footnotetext{
*bartosz.regula@gmail.com

†nvarun@ntu.edu.sg

\#buscemi@i.nagoya-u.ac.jp

§mgu@quantumcomplexity.org
}

Published by the American Physical Society under the terms of the Creative Commons Attribution 4.0 International license. Further distribution of this work must maintain attribution to the author(s) and the published article's title, journal citation, and DOI. measurements, making them cheap and easy to implement in the resource-theoretic setting; the strictly incoherent operations (SIO) $[4,11]$ allow for a similar implementation with incoherent ancillae, but require arbitrary measurements. Regrettably, these operations were found to be too limited in their operational capabilities $[13,25,26]$, suggesting that any nontrivial resource theory of coherence would require a larger set of allowed maps in order to be practically useful. Common choices of such larger sets, however, are often too permissive and lack a physical justification [7].

The question of "freeness" of operations within the resource theory of coherence has also been approached from a different perspective, by characterizing the nonclassicality of the channels themselves-first in a very general manner [27] and later with explicit applications to coherence theory $[11,14,22]$. These works suggest that the most important feature distinguishing truly nonclassical channels is their ability to detect the coherence present in a system and use it to manipulate the state, and so any class of channels which is to be considered free must necessarily be incapable of coherence detection. Although the study of maps based on various types of coherence nondetecting properties has attracted significant attention in recent years $[7,8,11,14,17-19,21,22]$, the limits of their power remain undiscovered.

In this work, we explore the limitations imposed on coherence manipulation by the inability of the free operations to create and detect coherence. We first study the class of dephasing-covariant incoherent operations (DIO) [7,8], constituting the largest class of operations that do not detect the coherence of any input state. We establish a complete description of pure-state transformations under these operations by relating them with the theory of majorization, also revealing an operational connection between DIO and other classes of free operations. To investigate the ultimate operational limits 
of coherence nondetecting channels, we then introduce the class of operations $\rho$-DIO, which are tailored to a specific input state and extend the class DIO. In order to understand and compare their strength, we quantify the capabilities of $\rho$-DIO in the fundamental operational tasks of coherence distillation and dilution. We show in particular that such maps satisfy a curious property: even though $\rho$-DIO cannot detect the coherence of the state $\rho$, they can still distill more coherence from $\rho$ than the class DIO; however, this advantage disappears in the one-shot task of dilution as well as generally in the asymptotic limit, where DIO match the capabilities of $\rho$-DIO in all state transformations. Our results give insight into the precise limits on the operational power of free operations which do not use coherence in manipulating coherence as a resource.

\section{DIO AND $\rho$-DIO}

We study quantum coherence as a basis-dependent concept. We will therefore fix an orthonormal basis $\{|i\rangle\}_{i=1}^{d}$ which we deem incoherent, and use $\mathcal{I}$ to denote the set of all states diagonal (incoherent) in this basis. We will use $\Delta(\cdot)=$ $\sum_{i}|i\rangle\langle i|\cdot| i\rangle\langle i|$ to denote the completely dephasing channel in this basis.

As discussed previously, it is natural to impose two conditions for a set of operations to be free: one is its inability to create coherence, in the sense that $\sigma \in \mathcal{I} \Rightarrow \Lambda(\sigma) \in \mathcal{I}$, and the other is its inability to detect coherence, in the sense that measurement statistics under any incoherent measurement after an operation $\Lambda$ should remain the same regardless of whether or not the input state possessed any coherence; precisely, $\langle i|\Lambda(\rho)| i\rangle=\langle i|\Lambda(\Delta(\rho))| i\rangle$ for all $i$. Imposing only the first constraint leads to the class of maximally incoherent operations (MIO) [2], which can exhibit undesirable properties such as being able to increase the number of levels of a pure state which are in superposition [12]. The class of channels which satisfies both constraints for all input states is precisely DIO, equivalently defined to commute with the dephasing channel: $\Lambda \circ \Delta(\rho)=\Delta \circ \Lambda(\rho) \forall \rho$. These operations have previously been considered in various contexts [7,8,14,27], and indeed they admit several interpretations. The maps DIO can be regarded as inherently classical [22,27], as any classical (incoherent) observer is unable to distinguish $\Lambda(\rho)$ from $\Lambda \circ \Delta(\rho)$, and hence is unable to determine whether the coherence of $\rho$ has been employed in the process. The latter point shows that DIO can also be understood as the operations which do not use coherence [11,14], as the properties of the output system accessible to a classical observer are independent of the coherence of the input.

The ability to detect coherence is of particular importance in practical setups relying on quantum coherence, such as interferometric experiments [11,22,28]. A general interferometric protocol can be understood as consisting of three separate parts: first, a state in superposition is created; second, path-dependent phases are encoded in the state with suitable unitary operations; and third, the information about the paths is extracted in a measurement. It is then explicit that the ability to create (in the first step) and detect (in the last step) coherence is crucial for any such setup to work, and indeed any operation which can neither create nor detect coherence is inherently free and cannot be used in such an experimental protocol.

However, consider now a scenario in which the input coherent state $\rho$ of a protocol is known: the operations which cannot detect the coherence of the input state are then precisely those which satisfy $\Lambda \circ \Delta(\rho)=\Delta \circ \Lambda(\rho)$ for this choice of $\rho$, and indeed it is not necessary to impose dephasing covariance for all inputs if one is concerned with acting on $\rho$ specifically. This point of view motivates us to define the class of $\rho$-dephasing-covariant incoherent operations $(\rho$ DIO), which we take to be the operations which commute with the dephasing channel $\Delta$ for a given input state $\rho$, and which therefore incorporate the ultimate limitations caused by the inability to detect or use the coherence of a particular input state.

It is clear that a $\rho$-DIO map can, in principle, create or detect coherence when acting on an input state other than $\rho$. However, the definition of $\rho$-DIO is justified whenever one deals with an explicit protocol which transforms a fixed input state to some desired output. Two such protocols form the foundations of the manipulation of coherence as a quantum resource: these are the tasks of coherence distillation [4,18,24], which aims to convert a given input state to a maximally coherent state, and coherence dilution $[4,17]$, which performs the opposite transformation of a maximally coherent input state to some desired state. The definition of $\rho$-DIO then motivates the following question: can the operational capabilities of DIO be surpassed by operations which nevertheless do not detect the coherence of the input state $\rho$ ? To address this question, we first describe the transformations achievable under DIO and later investigate whether $\rho$-DIO can outperform the former.

\section{PURE-STATE TRANSFORMATIONS UNDER DIO}

Although a fundamental and operationally meaningful choice of operations, the class DIO remains relatively unexplored and few of its properties are known. Other sets of operations are better understood: in particular, it is known that the transformations of pure states under the classes of incoherent operations (IO) [3] and strictly incoherent operations (SIO) $[4,11]$ are governed by majorization theory, in a manner similar to the manipulation of pure-state entanglement under local operations and classical communication [29]. Precisely, one has that a pure-state transformation $|\psi\rangle=$ $\sum_{i} \psi_{i}|i\rangle \rightarrow|\phi\rangle=\sum_{i} \phi_{i}|i\rangle$ is achievable under IO or SIO if and only if $\Delta(\psi) \prec \Delta(\phi)[12,30,31]$, i.e., if $\sum_{i=1}^{k}\left|\psi_{i}\right|^{2} \leqslant$ $\sum_{i=1}^{k}\left|\phi_{i}\right|^{2} \forall k \in\{1, \ldots, d\}$, where we assume that the coefficients of the states are arranged so that $\left|\psi_{1}\right| \geqslant \cdots \geqslant\left|\psi_{d}\right|$. Our first contribution is to extend this relation to the class DIO.

Theorem 1. The deterministic pure-state transformation $\psi \rightarrow \phi$ is possible under DIO if and only if $\Delta(\psi) \prec \Delta(\phi)$.

We refer to Appendix A for the full proof of the theorem. This establishes DIO as another class of operations in which pure-state transformations are fully governed by majorization theory, and reveals an operational equivalence between DIO, $\mathrm{IO}$, and SIO in manipulating pure states. The equivalence is nontrivial: the class DIO is incomparable with IO [12], and there exist coherence monotones which can increase under 
DIO despite always decreasing under the action of SIO and IO [32].

The theorem immediately lets us apply a plethora of results to coherence manipulation under DIO. For instance, the recent investigation of moderate-deviation interconversion rates under majorization in Refs. [33,34] allows one to precisely characterize DIO transformations beyond the single-shot regime; similarly, a recent investigation of quantum coherence fluctuation relations [35] relies purely on the theory of majorization, and our result immediately establishes that the results can be directly applied to describe the fluctuations and batteryassisted transformations under DIO.

The result can also be extended to so-called heralded probabilistic transformations, where a state $|\psi\rangle$ is transformed to one of the states $\left\{\left|\phi_{j}\right\rangle\right\}$ with a corresponding probability $p_{j}$, and the final state is identified unambiguously by a classical flag register; formally, the output states take the form $\phi_{j} \otimes$ $|j\rangle\langle j|$. One can similarly show the following.

Proposition 1. There exists a DIO effecting the transformation $\psi \rightarrow \sum_{j} p_{j} \phi_{j} \otimes|j\rangle\langle j|$ if and only if

$$
\Delta(\psi) \prec \sum_{j} p_{j} \Delta\left(\phi_{j}\right) .
$$

We refer the reader to the appendices for details. This again establishes an equivalence between DIO, IO, and SIO in such transformations, extending earlier partial results [21].

\section{COHERENCE MANIPULATION WITH $\rho$-DIO}

The existence of a $\rho$-DIO transformation between states $\rho$ and $\sigma$ is equivalent to the existence of a quantum channel $\Lambda$ such that $\Lambda(\rho)=\sigma$ and $\Lambda(\Delta(\rho))=\Lambda(\Delta(\sigma))$. This has strong connections with the concept of relative majorization and the distinguishability between the states $\rho$ and $\Delta(\rho)$ [36-38], and may at first sight suggest that majorization will also play a role in $\rho$-DIO transformations, making them no more powerful than DIO. We will show that this is, in fact, not the case. To investigate this problem, we now focus on the fundamental tasks of distillation and dilution.

\section{A. Distillation}

The $\varepsilon$-error one-shot distillable coherence under the class $\rho$-DIO is defined to be the largest size of the maximally coherent state $\left|\Psi_{m}\right\rangle=\sum_{i} \frac{1}{\sqrt{m}}|i\rangle$ reachable to within an error $\varepsilon$ under a single $\rho$-DIO transformation; formally, we have

$$
\begin{aligned}
& C_{d, \rho-\mathrm{DIO}}^{(1), \varepsilon}(\rho) \\
& \quad:=\log \max \left\{m \mid \max _{\Lambda \in \rho-\mathrm{DIO}} F\left(\Lambda(\rho), \Psi_{m}\right) \geqslant 1-\varepsilon\right\},
\end{aligned}
$$

where $F(\rho, \sigma)=\|\sqrt{\rho} \sqrt{\sigma}\|_{1}^{2}$ is the fidelity and the logarithm is base 2. Our first result exactly characterizes this quantity in terms of the hypothesis testing relative entropy $D_{H}^{\varepsilon}$, defined as [39-41]

$$
\begin{aligned}
& D_{H}^{\varepsilon}(\rho \| \sigma) \\
& \quad:=-\log \min \{\operatorname{Tr} M \sigma \mid 0 \leqslant M \leqslant \mathbb{1}, 1-\operatorname{Tr} M \rho \leqslant \varepsilon\} .
\end{aligned}
$$

This quantity finds use in the fundamental task of quantum hypothesis testing $[42,43]$, where one is interested in distinguishing between two quantum states $\rho$ and $\sigma$ by a measurement $\{M, \mathbb{1}-M\}$, with $D_{H}^{\varepsilon}(\rho \| \sigma)$ exactly quantifying the smallest probability of incorrectly accepting the hypothesis of possessing state $\rho$ as true $(\operatorname{Tr} M \sigma)$ while constraining the probability of incorrectly accepting the hypothesis of being in possession of state $\sigma$ as true $[\operatorname{Tr}(\mathbb{1}-M) \rho]$ to be, at most, $\varepsilon$. We remark that $D_{H}^{\varepsilon}(\rho \| \sigma)$ is efficiently computable as a semidefinite program [44].

We relate the hypothesis testing relative entropy with distillation in the following.

Theorem 2. The $\varepsilon$-error one-shot distillable coherence un$\operatorname{der} \rho$-DIO for any input state $\rho$ is given by

$$
C_{d, \rho-\mathrm{DIO}}^{(1), \varepsilon}(\rho)=\left\lfloor D_{H}^{\varepsilon}(\rho \| \Delta(\rho))\right\rfloor_{\log },
$$

where $\lfloor x\rfloor_{\log }:=\log \left\lfloor 2^{x}\right\rfloor$.

This formally establishes a property of the class of operations $\rho$-DIO that one might intuitively expect: the more distinguishable a state $\rho$ is from its dephased version $\Delta(\rho)$, the more coherence we can extract from it using $\rho$-DIO. This is indeed very natural in the framework of quantum coherence, as it gives explicit operational meaning to the information contained in the off-diagonal elements of the density matrix $\rho$. It is instructive to compare Eq. (2) with the expression for one-shot distillable coherence under DIO [18], where $D_{H}^{\varepsilon}$ additionally has to be optimized over a set of operators and does not enjoy an exact interpretation in this context.

Of particular importance will be the case $\varepsilon=0$, that is, exact deterministic distillation of coherence. The result then reduces to

$$
C_{d, \rho-\mathrm{DIO}}^{(1), 0}(\rho)=\left\lfloor D_{H}^{0}(\rho \| \Delta(\rho))\right\rfloor_{\log }=\log \left\lfloor\frac{1}{\operatorname{Tr} \Pi_{\rho} \Delta(\rho)}\right\rfloor,
$$

where $\Pi_{\rho}$ is the projection onto the support of $\rho$. In particular, combining the results of Theorems 1 and 2, we have the following.

Corollary 1. A pure state $|\psi\rangle=\sum_{i} \psi_{i}|i\rangle$ can be deterministically transformed to $\left|\Psi_{m}\right\rangle$ under DIO iff

$$
\max _{i}\left|\psi_{i}\right|^{2} \leqslant \frac{1}{m}
$$

while the transformation is possible under $\psi$-DIO iff

$$
\langle\psi|\Delta(\psi)| \psi\rangle=\sum_{i}\left|\psi_{i}\right|^{4} \leqslant \frac{1}{m} .
$$

A detailed derivation can be found in the appendices. The above allows us to easily construct examples of states such that even though $|\psi\rangle \rightarrow\left|\Psi_{m}\right\rangle$ is impossible under DIO, the transformation can be achieved by $\psi$-DIO. Consider, for example, the state $|\psi\rangle:=\left(\sqrt{\frac{5}{8}}, \sqrt{\frac{3}{16}}, \sqrt{\frac{3}{16}}\right)^{T}$, for which it can be verified that $\Delta\left(\Psi_{2}\right) \nsucc \Delta(\psi)$, which means the transformation $|\psi\rangle \rightarrow\left|\Psi_{2}\right\rangle$ is impossible by DIO (and, in fact, by all MIO [18]). However, we easily compute $\sum_{i}\left|\psi_{i}\right|^{4}=$ $\frac{59}{128}<\frac{1}{2}$, so $C_{d, \psi-\mathrm{DIO}}^{(1), 0}(\psi)=1$ and hence one coherence bit $\Psi_{2}$ can be exactly distilled. This explicitly shows an operational advantage provided by the operations $\rho$-DIO over DIO in state transformations and, in particular, in coherence distillation. Such an advantage is rather surprising: to any classical observer, the distillation protocol applied to the state $\rho$ is 
indistinguishable from a classical operation, yet it can distill more coherence than DIO or even the powerful class MIO. Furthermore, due to the majorization condition of Theorem 1, it is easy to see that the exact distillation of coherence under DIO cannot be enhanced by the use of a catalyst - that is, $\psi \otimes \phi \rightarrow \Psi_{m} \otimes \phi$ is possible under DIO if and only if $\psi \rightarrow$ $\Psi_{m}$ is possible-which then shows that $\rho$-DIO has strictly larger capabilities than even catalysis-assisted DIO.

However, now consider the many-copy scenario in which we can perform joint quantum operations on the composite system $\rho^{\otimes n}$. In the asymptotic limit of independent and identically distributed (i.i.d.) systems, one can then define the distillable coherence under $\rho$-DIO as

$$
C_{d, \rho-\mathrm{DIO}}^{\infty}(\rho)=\lim _{\varepsilon \rightarrow 0} \lim _{n \rightarrow \infty} \frac{1}{n} C_{d, \rho^{\otimes n}-\mathrm{DIO}}^{(1), \varepsilon}\left(\rho^{\otimes n}\right) .
$$

A simple application of Theorem 1 together with the quantum Stein's lemma $[45,46]$ reveals that we have, in fact, $C_{d, \rho-\mathrm{DIO}}^{\infty}(\rho)=D(\rho \| \Delta(\rho))$, that is, the relative entropy of coherence $D(\rho \| \Delta(\rho))$ characterizes the asymptotic rate of coherence distillation under $\rho$-DIO. But it is already known that under DIO, we also have $C_{d, \text { DIO }}^{\infty}(\rho)=D(\rho \| \Delta(\rho))[18,19]$, which means that $\rho$-DIO do not perform any better than DIO in the asymptotic limit. Taking into consideration the operational gap between the operations DIO and $\rho$-DIO in single-shot transformations, the asymptotic result can be quite surprising since it effectively shows that the advantage provided by $\rho$-DIO over DIO will be relatively minor and will disappear completely at the asymptotic level.

Finally, one can define the zero-error distillable coherence as

$$
C_{d, \rho-\mathrm{DIO}}^{\infty, 0}(\rho)=\lim _{n \rightarrow \infty} \frac{1}{n} C_{d, \rho^{\otimes n}-\mathrm{DIO}}^{(1), 0}\left(\rho^{\otimes n}\right) .
$$

Noting the additivity of $D_{H}^{0}(\rho \| \Delta(\rho))$, from Eq. (3) we immediately get that $C_{d, \rho-\mathrm{DIO}}^{\infty, 0}(\rho)=-\log \operatorname{Tr} \Pi_{\rho} \Delta(\rho)$.

\section{B. Dilution}

Consider the transformation of a maximally coherent state $\Psi_{m}$ into a general state $\rho$, using a $\Psi_{m}$-DIO protocol. The oneshot coherence cost is given by

$$
\begin{aligned}
& C_{c, \Psi_{m}-\mathrm{DIO}}^{(1), \varepsilon}(\rho) \\
& \quad:=\log \min \left\{m \mid \max _{\Lambda \in \Psi_{m}-\mathrm{DIO}} F\left(\Lambda\left(\Psi_{m}\right), \rho\right) \geqslant 1-\varepsilon\right\} .
\end{aligned}
$$

To characterize this quantity, we will consider the coherence monotone based on the max-relative entropy between $\rho$ and $\Delta(\rho)[12]$, given by

$$
R_{\Delta}(\rho):=\min \{\lambda \mid \rho \leqslant(1+\lambda) \Delta(\rho)\} .
$$

It is easy to verify that $R_{\Delta}(\Lambda(\rho)) \leqslant R_{\Delta}(\rho)$ for any $\rho$-DIO operation $\Lambda$. Using this quantity, we have the following.

Theorem 3. The $\varepsilon$-error one-shot coherence cost under $\Psi_{m^{-}}$ $\mathrm{DIO}$ is given by

$$
\begin{aligned}
& C_{c, \Psi_{m}-\mathrm{DIO}}^{(1), \varepsilon}(\rho) \\
& \quad=\log \left\lceil\min \left\{R_{\Delta}(\omega)+1 \mid \omega \in \mathbb{D}, F(\rho, \omega) \geqslant 1-\varepsilon\right\}\right\rceil,
\end{aligned}
$$

where $\mathbb{D}$ denotes the set of all density matrices.
Interestingly, comparing the above with the results obtained previously for DIO [17], we have that

$$
C_{c, \mathrm{DIO}}^{(1), \varepsilon}(\rho)=C_{c, \Psi_{m}-\mathrm{DIO}}^{(1), \varepsilon}(\rho)
$$

that is, the operations $\Psi_{m}$-DIO provide no advantage over DIO whatsoever. Combining this with the fact that the asymptotic coherence cost under DIO is exactly given by $D(\rho \| \Delta(\rho))$ [19], we similarly have that

$$
C_{d, \Psi_{m}-\mathrm{DIO}}^{\infty}(\rho)=\lim _{\varepsilon \rightarrow 0} \lim _{n \rightarrow \infty} \frac{1}{n} C_{c, \Psi_{m}-\mathrm{DIO}}^{(1), \varepsilon}\left(\rho^{\otimes n}\right)=D(\rho \| \Delta(\rho)) .
$$

We can also note that the zero-error coherence cost under $\Psi_{m}$-DIO (or DIO) is exactly given by $\log \left\lceil R_{\Delta}(\rho)+1\right\rceil$, and noticing the multiplicativity of $R_{\Delta}+1$, one can see that the asymptotic zero-error cost of coherence dilution will be simply given by $\log \left[R_{\Delta}(\rho)+1\right]$.

\section{General transformations and monotones}

When discussing asymptotic state transformations, one is in particular interested in the largest rate $R(\rho \rightarrow \sigma)$ at which copies of $\rho$ can be transformed to copies of $\sigma$ under the given class of operations. Our results can be used to show that the rate of any such transformation under $\rho$-DIO is completely characterized by the relative entropy between the states and their diagonals.

To see this, notice first that any transformation $\rho \rightarrow \omega \rightarrow$ $\sigma$ such that the operation taking $\rho$ to $\omega$ is $\rho$-DIO and the operation taking $\omega$ to $\sigma$ is $\omega$-DIO results in an overall protocol $\rho \rightarrow \sigma$, which is $\rho$-DIO. This allows us to employ maximally coherent states $\Psi_{m}$ as an intermediary in coherence transformations. Using the fact that $\Psi_{2^{m}}=|+\rangle\left\langle+\left.\right|^{\otimes m}\right.$, we can interpret the distillable coherence $C_{d, \rho-\mathrm{DIO}}^{\infty}(\rho)$ as the rate $R(\rho \rightarrow|+\rangle\langle+|)$ under $\rho$-DIO, and the coherence cost $C_{c, \Psi_{m}-\mathrm{DIO}}^{\infty}(\sigma)$ as the rate $1 / R(|+\rangle\langle+| \rightarrow \sigma)$ under $|+\rangle\langle+|-$ DIO; a straightforward argument in analogy with Ref. [47] then shows the following.

Corollary 2. For any states $\rho$ and $\sigma$, the maximal rate of the asymptotic transformation $\rho \rightarrow \sigma$ under $\rho$-DIO operations is given by

$$
R(\rho \rightarrow \sigma)=\frac{D(\rho \| \Delta(\rho))}{D(\sigma \| \Delta(\sigma))} .
$$

As this is also true for DIO [19], asymptotically, $\rho$-DIO provide no advantage whatsoever over DIO in any state transformation.

Furthermore, one can obtain various useful sufficient conditions for the transformations in the one-shot setting. For instance, we show that the monotone $R_{\Delta}$ can also be used to characterize state transformations under $\rho$-DIO which go beyond coherence distillation and dilution.

Proposition 2. If $R_{\Delta}(\sigma)+1 \leqslant 1 / \operatorname{Tr} \Pi_{\rho} \Delta(\rho)$, then there exists a $\rho$-DIO map such that $\Lambda(\rho)=\sigma$.

In the particularly simple case of single-qubit transformations, we furthermore establish an equivalence of $\rho$-DIO and DIO.

Proposition 3. For any single-qubit states $\rho$ and $\sigma$, the transformation $\rho \rightarrow \sigma$ is possible under $\rho$-DIO if and only if it is possible under DIO, which holds if and only if [12] $R_{\Delta}(\rho) \geqslant R_{\Delta}(\sigma)$ and $\|\rho\|_{\ell_{1}} \geqslant\|\sigma\|_{\ell_{1}}$. 
Here, $\|\rho\|_{\ell_{1}}=\sum_{i, j}|\langle i|\rho| j\rangle|$. We note from Ref. [12] that single-qubit DIO transformations have been shown to be equivalent to both MIO and SIO transformations, and our result thus extends this equivalence to $\rho$-DIO also. This does not hold beyond dimension 2, as we have demonstrated in Sec. IV A a transformation from a qutrit to a qubit system achievable with $\rho$-DIO, but impossible under MIO and DIO.

Necessary conditions for single-shot $\rho$-DIO transformations can be characterized by monotones under this class, i.e., functions which obey the property that if there exists a transformation $\rho \rightarrow \sigma$ under $\rho$-DIO, then $f(\rho) \geqslant f(\sigma)$. Some DIO monotones discussed, e.g., in Ref. [12], will in fact also be $\rho$-DIO monotones-this includes $R_{\Delta}$ or the relative entropy $D(\rho \| \Delta(\rho))$. Indeed, any divergence which satisfies the data-processing inequality will form a $\rho$-DIO monotone. Importantly, this includes Rényi relative entropies $D_{\alpha}(\rho \| \Delta(\rho))=\frac{1}{\alpha-1} \log \operatorname{Tr} \rho^{\alpha} \Delta(\rho)^{1-\alpha}$, but only in the range $\alpha \in[0,2]$ [48], which contrasts with the set DIO for which all $\alpha$ give a valid monotone [12]. For a pure state, the Rényi relative entropies reduce to $D_{\alpha}(\psi \| \Delta(\psi))=S_{2-\alpha}(\psi)$ [12], where $S_{\gamma}(\psi)=\frac{\gamma}{1-\gamma} \log \|\Delta(\psi)\|_{\ell_{\gamma}}$ are the Rényi entropies. This shows, in particular, that $\ell_{p}$ norms of the squared moduli of the coefficients of a pure state are $\psi$-DIO monotones for $p$ in the range $p \in[0,1]$ and reverse monotones for $p \in[1,2]$. An outstanding question is whether such monotones form a complete set, in the sense that the inequality $f(\rho) \geqslant f(\sigma)$ for each monotone $f$ implies that there exists a $\rho$-DIO transformation taking $\rho$ to $\sigma$. Although a complete set of infinitely many monotones can be defined [49-51], it is unclear if there exists a finite set of conditions fully characterizing transformations under $\rho$-DIO.

\section{DISCUSSION}

In this work, we tackled the fundamental question of how to efficiently manipulate the resource of quantum coherence under operations which do not use coherence and thus, to a classical observer, appear classical. We studied this question under two classes of channels: DIO, respecting dephasing covariance for any input state, and $\rho$-DIO, tailored to a specific input. We first shed light on the operational power of DIO and explicitly characterized pure-state transformations under this class, revealing a relation between DIO and majorization theory and thereby connecting DIO to several other classes of free operations. To push the characterization of coherence manipulation under coherence nondetecting operations to its very limit, we introduced the class of operations $\rho$-DIO and investigated the advantages that this extension provides. We showed, in particular, that even though the coherence of the input state is not detected, $\rho$-DIO allow for transformations impossible under DIO: they can distill more coherence than DIO in the one-shot setting. Despite $\rho$-DIO constituting a significant relaxation of the constraints of DIO, the increased capabilities of such channels are limited to nonasymptotic regimes-we showed the advantages to disappear completely at the asymptotic level, where both sets of operations achieve the same performance in all transformations. This suggests that the simpler class $\rho$-DIO closely approximates the performance of all DIO and no significant operational advantage can be obtained by tailoring the coherence nondetecting restriction to a specific input. The results provide insight into the structure of the ultimate physical constraints on coherence manipulation with free operations, and establish connections in the operational description of quantum coherence by quantitatively relating coherence manipulation to the distinguishability between a state $\rho$ and its dephased version $\Delta(\rho)$.

Note added. Recently, Wang and Wilde [52] as well as Buscemi et al. [53] studied the distinguishability of pairs of states in an operational setting and independently obtained results which overlap with parts of this manuscript. Note, however, that although our characterization of $\rho$-DIO relies precisely on the distinguishability between $\rho$ and $\Delta(\rho)$, the tasks of coherence distillation and dilution studied herein are different from the operational framework of Ref. [52] concerned with manipulating distinguishability.

\section{ACKNOWLEDGMENTS}

We acknowledge discussions with Ludovico Lami. This work was supported by the National Research Foundation of Singapore Fellowship No. NRF-NRFF2016-02, the National Research Foundation and L'Agence Nationale de la Recherche joint Project No. NRF2017-NRFANR004 VanQuTe, the program for FRIAS-Nagoya IAR Joint Project Group, and the Japan Society for the Promotion of Science (JSPS) KAKENHI Grant No. 19H04066.

\section{APPENDIX A: PURE-STATE TRANSFORMATIONS UNDER DIO}

Let $\{|x\rangle\}_{x=1 \ldots d_{\text {in }}} \subset \mathcal{H}_{\text {in }}$ and $\{|y\rangle\}_{y=1 \ldots d_{\text {out }}} \subset \mathcal{H}_{\text {out }}$ be the incoherent bases on the input and output Hilbert spaces, respectively.

Definition 1. DIO: A channel $\mathcal{E}: \mathcal{L}\left(\mathcal{H}_{\text {in }}\right) \rightarrow \mathcal{L}\left(\mathcal{H}_{\text {out }}\right)$ is a dephasing- covariant incoherent operation (DIO) if $\Delta \circ \mathcal{E}=$ $\mathcal{E} \circ \Delta$, where $\Delta(\cdot)$ is the dephasing channel with respect to the incoherent basis on the corresponding system.

We now cast the DIO property of a channel in terms of its Kraus operator representations. Let $\mathcal{E}(\cdot)=\sum_{i=1}^{n} K_{i}(\cdot) K_{i}^{\dagger}$; we do not have to worry about the value of $n$. The equivalence of $\Delta \circ \mathcal{E}$ and $\mathcal{E} \circ \Delta$ can be translated to the equality of their Choi operators:

$$
\begin{aligned}
\mathrm{id} & \otimes[\Delta \circ \mathcal{E}]\left(\sum_{x_{1}, x_{2}}\left|x_{1} x_{1}\right\rangle\left\langle x_{2} x_{2}\right|\right) \\
& =\mathrm{id} \otimes[\mathcal{E} \circ \Delta]\left(\sum_{x_{1}, x_{2}}\left|x_{1} x_{1}\right\rangle\left\langle x_{2} x_{2}\right|\right) \\
& \Rightarrow \sum_{i, x_{1}, x_{2}, y}\left|x_{1}\right\rangle\left\langle x_{2}\right| \otimes\left(|y\rangle\left\langle y\left|K_{i}\right| x_{1}\right\rangle\left\langle x_{2}\left|K_{i}^{\dagger}\right| y\right\rangle\langle y|\right) \\
& =\sum_{i, x}|x\rangle\langle x| \otimes\left(K_{i}|x\rangle\langle x| K_{i}^{\dagger}\right) .
\end{aligned}
$$

This leads to the following handy properties of any Kraus operator representation of a DIO:

Observation 1. Define the vectors $\mathbf{K}(y, x) \in \mathcal{V} \equiv \mathbb{C}^{n}$, $d_{\text {in }} d_{\text {out }}$ in number as follows:

$$
\mathbf{K}(y, x):=\left(\left\langle y\left|K_{1}\right| x\right\rangle,\left\langle y\left|K_{2}\right| x\right\rangle, \ldots,\left\langle y\left|K_{n}\right| x\right\rangle\right) .
$$


Also define $S_{y \mid x}:=\langle\mathbf{K}(y, x), \mathbf{K}(y, x)\rangle$, where $\langle\cdot, \cdot\rangle$ is the standard Hermitian inner product on $\mathbb{C}^{n}$. Then, the following conditions together capture the DIO property of the completely positive $(\mathrm{CP}) \operatorname{map} \mathcal{E}(\cdot)=\sum_{i=1}^{n} K_{i}(\cdot) K_{i}^{\dagger}$ :

(1) Diagonal (i.e., incoherent) input produces diagonal output on average: $\left\langle\mathbf{K}(y, x), \mathbf{K}\left(y_{1}, x\right)\right\rangle=S_{y \mid x} \delta_{y y_{1}}$.

(2) Diagonal- free input produces diagonal- free output on average: $\left\langle\mathbf{K}(y, x), \mathbf{K}\left(y, x_{1}\right)\right\rangle=S_{y \mid x} \delta_{x x_{1}}$.

(3) Trace is preserved: $\sum_{y=1}^{d_{\text {out }}} S_{y \mid x}=1$ for all $x \in$, $\left\{1,2 \ldots, d_{\text {in }}\right\}$; in other words, the matrix $S$ with components $S_{y x}:=S_{y \mid x}$ is column stochastic (justifying the "conditional" notation).

It is important to bear in mind that each of these conditions involves summing over all the $n$ Kraus operators. In particular, if the "on-average" condition (1) was tightened to apply to each Kraus operator separately while removing condition (2) altogether, the resulting conditions would characterize the class of incoherent operations (IO). Likewise, if both (1) and (2) were tightened to apply to each Kraus operator, we would have strictly incoherent operations (SIO).

For convenience, we also define the normalized vectors $\hat{\boldsymbol{\kappa}}(y, x):=\frac{1}{\sqrt{S_{y \mid x}}} \mathbf{K}(y, x)$, leading to $\left\langle\hat{\boldsymbol{\kappa}}(y, x), \hat{\boldsymbol{\kappa}}\left(y_{1}, x\right)\right\rangle=\delta_{y y_{1}}$ and $\left\langle\hat{\kappa}(y, x), \hat{\kappa}\left(y, x_{1}\right)\right\rangle=\delta_{x x_{1}}$. In the cases where $S_{y \mid x}=0$, just define $\hat{\kappa}(y, x)$ to be some unit vector orthogonal to the rest, suitably expanding the space.

\section{Deterministic pure- to- pure state conversion}

Now consider the problem of determining the conditions under which there is a DIO deterministically mapping a given pure state $\psi$ to another, $\phi$. We shall prove the following theorem. Say the DIO given by $\mathbf{K}(y, x)$ achieves the desired transformation. Then,

Theorem 4. A given initial state $|\psi\rangle=\sum_{x} \mu_{x}|x\rangle$ can be mapped deterministically to a given target state $|\phi\rangle=v_{y}|y\rangle$ by a DIO if and only if the majorization relation

$$
\mathbf{p} \prec \mathbf{q}
$$

holds, where $p_{x}:=\left|\mu_{x}\right|^{2}$ and $q_{y}:=\left|v_{y}\right|^{2}$.

Remark 1. Note on majorization. For a vector $\mathbf{v}$ on a finite- dimensional real vector space, define $\mathbf{v}^{\downarrow}$ as the vector whose components are the components of $\mathbf{v}$ arranged in non increasing order. For example, $(2,-5,2,4)^{\downarrow}=(4,2,2,-5)$. For a pair of vectors $(\mathbf{u}, \mathbf{v})$, the majorization relation $\mathbf{u} \prec \mathbf{v}$ ("v majorizes $\mathbf{u}$ ") is then defined as the conjunction of the following conditions:

$$
\begin{gathered}
u_{1}^{\downarrow} \leqslant v_{1}^{\downarrow} ; \\
u_{1}^{\downarrow}+u_{2}^{\downarrow} \leqslant v_{1}^{\downarrow}+v_{2}^{\downarrow} ; \\
\vdots \\
u_{1}^{\downarrow}+u_{2}^{\downarrow} \cdots+u_{d}^{\downarrow} \leqslant v_{1}^{\downarrow}+v_{2}^{\downarrow} \cdots+v_{d}^{\downarrow},
\end{gathered}
$$

where $d$ is the larger of the dimensionalities of $u$ and $v$ (we append the shorter vector with a suitable number of trailing zeros).

A real matrix with non-negative entries is column stochastic (row stochastic) if each of its columns (rows) adds up to 1 ; a matrix that is both row and column stochastic is said to be bistochastic. A non-negative real matrix is sub- *stochastic if another non-negative matrix can be added to it to make it *stochastic ("**" can stand for "column-", "row-", "bi-", or the absence of any of these qualifiers).

For the purposes of proving the above theorem, we will use the following properties of majorization (see, e.g., [54]):

(1) For normalized probability vectors $\mathbf{u}$ and $\mathbf{v}$, the relation $\mathbf{u} \prec \mathbf{v}$ holds if and only if there exists a bistochastic matrix $T$ such that $\mathbf{u}=T \mathbf{v}$.

(2) For non-negative vectors $\mathbf{u}$ and $\mathbf{v}$, if there exists a subbistochastic matrix $T$ such that $\mathbf{u}=T \mathbf{v}$, then $\mathbf{v}$ is said to weakly majorize $\mathbf{u}$; weak majorization between normalized probability vectors implies nonweak majorization (i.e., the previous condition).

Proof. Since the overall output is the pure state $\phi$, the output of each individual Kraus operator must necessarily be proportional to $\phi$. In other words, there exists a normalized $\hat{\mathbf{c}} \in \mathbb{C}^{n}$ such that

$$
\sum_{x_{1}=1}^{d_{\text {in }}} K_{i}\left(y, x_{1}\right) \mu_{x_{1}}=c_{i} v_{y}
$$

$\forall i, y$. Multiplying both sides by $\overline{K_{i}(y, x)}$ and summing over $i$,

$$
\begin{aligned}
& \sum_{x_{1}=1}^{d_{\text {in }}} \mu_{x_{1}} \sum_{i=1}^{n} \overline{K_{i}(y, x)} K_{i}\left(y, x_{1}\right)=\sum_{i=1}^{n} \overline{K_{i}(y, x)} c_{i} v_{y} \\
& \Rightarrow \sum_{x_{1}=1}^{d_{\text {in }}} \mu_{x_{1}} S_{y \mid x} \delta_{x x_{1}}=\langle\mathbf{K}(y, x), \hat{\mathbf{c}}\rangle v_{y} \\
& \Rightarrow \mu_{x} \sqrt{S_{y \mid x}}=\langle\hat{\boldsymbol{\kappa}}(y, x), \hat{\mathbf{c}}\rangle v_{y} \\
& \Rightarrow p_{x} \equiv\left|\mu_{x}\right|^{2}=\left|\mu_{x}\right|^{2} \sum_{y=1}^{d_{\text {out }}} S_{y \mid x}=\sum_{y=1}^{d_{\text {out }}}|\langle\hat{\boldsymbol{\kappa}}(y, x), \hat{\mathbf{c}}\rangle|^{2}\left|v_{y}\right|^{2} \\
& \equiv \sum_{y=1}^{d_{\text {out }}}|\langle\hat{\boldsymbol{\kappa}}(y, x), \hat{\mathbf{c}}\rangle|^{2} q_{y} .
\end{aligned}
$$

The second line above follows from condition (2); the following line by dividing throughout by $\sqrt{S_{y \mid x}}$ and applying the definition of $\hat{\kappa}(y, x)$; in the last line, we just sum the previous line's expressions over $y$ and use the stochasticity of $S$.

Now define $T_{x \mid y}:=|\langle\hat{\boldsymbol{\kappa}}(y, x), \hat{\mathbf{c}}\rangle|^{2}$. The normalization of $\hat{\mathbf{c}}$ and orthonormality of $\{\hat{\boldsymbol{\kappa}}(y, x)\}_{x=1}^{d_{\text {in }}}$ (for each $y$ ) and $\{\hat{\boldsymbol{\kappa}}(y, x)\}_{y=1}^{d_{\text {out }}}$ (for each $x$ ) together imply that $T$ is sub- bistochastic. This implies that $\mathbf{p}$ is weakly majorized by $\mathbf{q}$; normalization of the distributions implies (nonweak) majorization. Incidentally, the same normalization arguments also imply that $n=d_{\text {in }}$ suffices.

Thus, majorization of the input coherence distribution by the output is necessary for the existence of a DIO deterministically mapping $\psi \mapsto \phi$. Since this condition is already known to be sufficient for the existence of such an SIO, its sufficiency for the existence of such a DIO follows.

\section{Probabilistic pure- to- pure state conversion}

A possible definition of probabilistic conversion of a given state $|\psi\rangle$ to an ensemble $\left\{\left(\eta_{j},\left|\phi_{j}\right\rangle\right)\right\}$ under a class of op- 
erations is one where a channel $\Lambda \equiv\left\{K_{j}\right\}$ belonging to the class satisfies $K_{j} \psi K_{j}^{\dagger}=\eta_{j} \phi_{j}$ (possibly with some of the $\phi_{j}$ mutual duplicates). This definition is not easily amenable to the treatment of the previous section, owing to the lack of individual Kraus operator-based constraints in DIO. This is in contrast with IO and SIO, whose definitions constrain each Kraus operator. Extension of our results to the pure- state- tomixed- state case is hindered by this obstacle.

Nevertheless, we can say something useful about heralded probabilistic conversion from a pure state $|\psi\rangle$ to an ensemble $\left\{\left(\eta_{j},\left|\phi_{j}\right\rangle\right)\right\}$. This entails that a conversion to $\phi_{j}$ be heralded by a correlated "flag" system whose state unambiguously identifies $j$. In other words, we require a DIO to map $\psi$ to $\sum_{j} \eta_{j} \sigma_{j} \otimes \phi_{j}$, with the "flag states" $\sigma_{j}$ on the first subsystem unambiguously distinguishable. We might as well set these to some mutually orthogonal $|j\rangle\langle j|$ without loss of generality. To keep the game fair, we shall require $|j\rangle$ to constitute the axiomatic incoherent basis for the flag system.

Proposition 4. There exists a DIO effecting the transformation $\psi \mapsto \sum_{j} \eta_{j}|j\rangle\langle j| \otimes \phi_{j}$ if and only if

$$
\mathbf{p} \prec \sum_{j} \eta_{j} \mathbf{q}_{j}^{\downarrow},
$$

where $\mathbf{p}$ is as before and $q_{y}^{j}:=\left|v_{y}^{j}\right|^{2}$ for $\left|\phi_{j}\right\rangle=\sum_{y} v_{y}^{j}|y\rangle$.

Proof. Without loss of generality, we can decompose the requisite DIO using Kraus operators of the form

$$
K_{j, m}=\sum_{x, y} K_{j, m}(j, y ; x)|j\rangle \otimes|y\rangle\langle x|,
$$

with

$$
\sum_{m} K_{j, m} \psi K_{j, m}^{\dagger}=\eta_{j}|j\rangle\langle j| \otimes \phi_{j} .
$$

Adapting the notation of the previous section, the DIO conditions can be cast as

(1) $\left\langle\mathbf{K}(j, y ; x), \mathbf{K}\left(j_{1}, y_{1} ; x\right)\right\rangle=S_{j, y \mid x} \delta_{j j_{1}} \delta_{y y_{1}}$.

(2) $\left\langle\mathbf{K}(j, y ; x), \mathbf{K}\left(j, y ; x_{1}\right)\right\rangle=S_{j, y \mid x} \delta_{x x_{1}}$.

(3) $\sum_{j, y} S_{j, y \mid x}=1$ for all $x$.

In fact, considering the form (A8) allows us to strengthen condition (2) above to

$$
\left\langle\mathbf{K}(j, y ; x), \mathbf{K}\left(j, y ; x_{1}\right)\right\rangle_{j}=S_{j, y \mid x} \delta_{x x_{1}},
$$

where $\langle\mathbf{u}, \mathbf{v}\rangle_{j}:=\sum_{m} \overline{u_{j, m}} v_{j, m}$ for $\mathbf{u}, \mathbf{v}$ in the abstract vector space $\mathcal{V}$ defined above. The rest of our proof to Theorem 1 can be applied as such, but with all such vectors restricted to the subspace corresponding to a specific $j$. This leads to

$$
p_{x} \sum_{y} S_{j, y \mid x}=\sum_{y}\left|\left\langle\hat{\boldsymbol{\kappa}}(j, y ; x), \mathbf{c}^{j}\right\rangle\right|^{2} q_{y}^{j},
$$

where

$$
K_{j, m}|\psi\rangle=c_{m}^{j}|j\rangle \otimes\left|\phi_{j}\right\rangle,
$$

with $\eta_{j}=\sum_{m}\left|c_{m}^{j}\right|^{2}$. Thus,

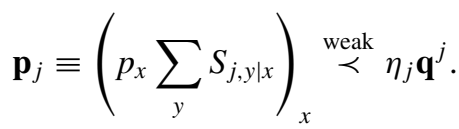

From the properties of the majorization relation, we have

$$
\mathbf{p}_{j}^{\downarrow} \stackrel{\text { weak }}{\prec} \eta_{j} \mathbf{q}_{j}^{\downarrow} .
$$

Summing over $j$ yields

$$
\sum_{j} \mathbf{p}_{j}^{\downarrow} \stackrel{\text { weak }}{\prec} \sum_{j} \eta_{j} \mathbf{q}_{j}^{\downarrow},
$$

which strengthens under normalization considerations to nonweak majorization. But, $\mathbf{p}=\sum_{j} \mathbf{p}_{j} \prec \sum_{j} \mathbf{p}_{j}^{\downarrow}$. Therefore,

$$
\mathbf{p} \prec \sum_{j} \eta_{j} \mathbf{q}_{j}^{\downarrow} .
$$

Again, the converse follows from the corresponding result about SIO.

\section{APPENDIX B: $\rho$-DIO TRANSFORMATIONS}

\section{Distillation}

Consider the rate of distillation of coherence under the operations $\rho$-DIO, i.e., channels $\Lambda$ such that $\Delta \circ \Lambda(\rho)=$ $\Lambda \circ \Delta(\rho)$ for some fixed $\rho$. We will use $\langle A, B\rangle=\operatorname{Tr}\left(A^{\dagger} B\right)$ for the Hilbert-Schmidt inner product.

Theorem 5. The one-shot distillable coherence under $\rho$ DIO for any input state $\rho$ is given by

$$
C_{d, \rho-\mathrm{DIO}}^{(1), \varepsilon}(\rho)=\left\lfloor D_{H}^{\varepsilon}(\rho \| \Delta(\rho))\right\rfloor_{\log },
$$

where $\lfloor x\rfloor_{\log }:=\log \left\lfloor 2^{x}\right\rfloor$.

Proof. The $\varepsilon$-error one-shot rate of distillation can be expressed as

$$
C_{d, \rho-\mathrm{DIO}}^{(1), \varepsilon}(\rho):=\log \max \left\{m \in \mathbb{N} \mid F_{\rho-\mathrm{DIO}}(\rho, m) \geqslant 1-\varepsilon\right\},
$$

where $F_{\rho-\text { DIO }}$ is the achievable fidelity of distillation, i.e.,

$$
F_{\rho-\mathrm{DIO}}(\rho, m):=\max _{\Lambda \in \rho-\mathrm{DIO}} F\left(\Lambda(\rho), \Psi_{m}\right),
$$

with $\Psi_{m}$ the $m$-dimensional maximally coherent state.

Defining the twirling $\mathcal{T}(\cdot)=\frac{1}{d !} \sum_{i=1}^{d !} U_{\pi(i)} \cdot U_{\pi(i)}^{\dagger}$, where each $\pi(i)$ is a permutation of the basis vectors, we have that

$$
\begin{aligned}
F_{\rho-\mathrm{DIO}}(\rho, m) & =\max _{\Lambda \in \rho-\mathrm{DIO}} F\left(\mathcal{T} \circ \Lambda(\rho), \mathcal{T}\left(\Psi_{m}\right)\right) \\
& =\max _{\Lambda \in \rho-\mathrm{DIO}} F\left(\mathcal{T} \circ \Lambda(\rho), \Psi_{m}\right),
\end{aligned}
$$

that is, it suffices to optimize over twirled maps of the form $\Lambda=\mathcal{T} \circ \Lambda$. Due to permutation invariance, the output of such a map must satisfy $\langle i|\Lambda(\cdot)| i\rangle=\langle\pi(i)|\Lambda(\cdot)| \pi(i)\rangle \forall i$ and $\langle i|\Lambda(\cdot)| j\rangle=\langle\pi(i)|\Lambda(\cdot)| \pi(j)\rangle \forall i \neq j$, where $\pi$ is an arbitrary permutation; imposing these constraints, we can write any such map as

$$
\Lambda(Q)=\langle X, Q\rangle \Psi_{m}+\langle\mathbb{1}-X, Q\rangle \frac{\mathbb{1}-\Psi_{m}}{m-1},
$$

for some operator $X$. The complete positivity of $\Lambda$ is equivalent to the condition $0 \leqslant X \leqslant \mathbb{1}$, and to further impose that 
$\Lambda \in \rho$-DIO, we need that

$$
\begin{aligned}
\frac{\mathbb{1}}{m} & =\Delta \circ \Lambda(\rho) \\
& =\Lambda \circ \Delta(\rho) \\
& =\langle X, \Delta(\rho)\rangle \Psi_{m}+(1-\langle X, \Delta(\rho))\rangle \frac{\mathbb{1}-\Psi_{m}}{m-1},
\end{aligned}
$$

which is satisfied if and only if $\langle X, \Delta(\rho)\rangle=\frac{1}{m}$. Altogether, we have

$$
\begin{aligned}
& F_{\rho-\mathrm{DIO}}(\rho, m) \\
& \quad=\max \left\{\langle X, \rho\rangle \mid 0 \leqslant X \leqslant \mathbb{1},\langle X, \Delta(\rho)\rangle=\frac{1}{m}\right\} .
\end{aligned}
$$

Although originally defined for $m \in \mathbb{N}$, we extend the definition of $F_{\rho \text {-DIO }}$ to any $m \geqslant 1$ as in Eq. (B6). The one-shot rate of distillation is then

$$
\begin{aligned}
& C_{d, \rho-\mathrm{DIO}}^{(1), \varepsilon}(\rho) \\
& \quad=\left\lfloor\log \max \left\{m \in \mathbb{R} \mid F_{\rho-\mathrm{DIO}}(\rho, m) \geqslant 1-\varepsilon\right\}\right\rfloor_{\log } \\
& \quad=\lfloor-\log \min \{\langle X, \Delta(\rho)\rangle \mid\langle X, \rho\rangle \geqslant 1-\varepsilon, 0 \leqslant X \leqslant \mathbb{1}\}\rfloor_{\log } \\
& \quad=\left\lfloor D_{H}^{\varepsilon}(\rho \| \Delta(\rho))\right\rfloor_{\log },
\end{aligned}
$$

where $D_{H}^{\varepsilon}$ is the hypothesis testing relative entropy.

By considering the asymptotic scenario, we then have

$$
\begin{aligned}
C_{d, \rho-\mathrm{DIO}}^{\infty}(\rho) & =\lim _{\varepsilon \rightarrow 0} \lim _{n \rightarrow \infty} \frac{1}{n} C_{d, \rho^{\otimes n}-\mathrm{DIO}}^{(1), \varepsilon}\left(\rho^{\otimes n}\right) \\
& =D(\rho \| \Delta(\rho)),
\end{aligned}
$$

by quantum Stein's lemma [45].

Recall from Ref. [18] that for DIO, we have

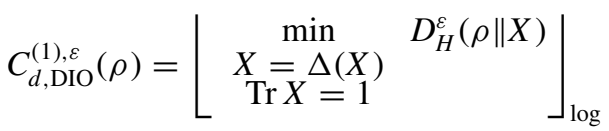

and

$$
C_{d, \mathrm{DIO}}^{\infty}(\rho)=D(\rho \| \Delta(\rho)),
$$

which shows that although $\rho$-DIO can have a larger rate of one-shot distillation than DIO, asymptotically the operations have the same capabilities in distillation.

\section{Exact distillation}

Now consider the case of zero-error distillation, that is, exact transformations $\rho \rightarrow \Psi_{m}$ under $\rho-$ DIO. From the above, we have that the rate of zero-error distillation is given by

$$
C_{d, \rho-\mathrm{DIO}}^{(1), 0}(\rho)=\left\lfloor D_{H}^{0}(\rho \| \Delta(\rho))\right\rfloor_{\log }=\log \left\lfloor\frac{1}{\left\langle\Pi_{\rho}, \Delta(\rho)\right\rangle}\right\rfloor,
$$

This simplifies in particular for the case of a pure state $|\psi\rangle=\sum_{i} \psi_{i}|i\rangle$ :

$$
\begin{aligned}
C_{d, \psi-\mathrm{DIO}}^{(1), 0}(\psi) & =\log \left\lfloor\langle\psi|\Delta(\psi)| \psi\rangle^{-1}\right\rfloor \\
& =\log \left\lfloor\left(\operatorname{Tr} \Delta(\psi)^{2}\right)^{-1}\right\rfloor \\
& =\log \left\lfloor\left(\sum_{i}\left|\psi_{i}\right|^{4}\right)^{-1}\right\rfloor .
\end{aligned}
$$

Explicitly, we have that

$$
\psi \rightarrow \Psi_{m} \Longleftrightarrow \sum_{i}\left|\psi_{i}\right|^{4} \leqslant \frac{1}{m} .
$$

Notice that the state $|\psi\rangle:=\left(\sqrt{\frac{5}{8}}, \sqrt{\frac{3}{16}}, \sqrt{\frac{3}{16}}\right)^{T}$ from the main text gives an explicit example of a case where coherence monotones under IO and DIO can increase in the $\rho$-DIO transformation: specifically, consider the monotone $C_{2}(|\psi\rangle)=$ $\sum_{i=2}^{d}\left|\psi_{i}^{\downarrow}\right|^{2}$, where $\psi_{i}^{\downarrow}$ denote coefficients of $|\psi\rangle$ arranged in nonincreasing order by magnitude $[31,55]$. For this monotone, with $|\psi\rangle$ as above, we have $C_{2}(|\psi\rangle)=\frac{3}{8}$ but $C_{2}(|+\rangle)=\frac{1}{2}$.

Finally, we remark the additivity of $-\log \operatorname{Tr} \Pi_{\rho} \Delta(\rho)$ in the sense that

$$
\begin{aligned}
-\log \left\langle\Pi_{\rho^{\otimes n}}, \Delta\left(\rho^{\otimes n}\right)\right\rangle & =-\log \left\langle\Pi_{\rho}^{\otimes n}, \Delta(\rho)^{\otimes n}\right\rangle \\
& =-n \log \left\langle\Pi_{\rho}, \Delta(\rho)\right\rangle,
\end{aligned}
$$

which, in particular, gives the asymptotic zero-error distillable coherence as

$$
C_{d, \rho-\mathrm{DIO}}^{\infty, 0}(\rho)=-\log \left\langle\Pi_{\rho}, \Delta(\rho)\right\rangle .
$$

\section{Dilution}

Theorem 6. The one-shot coherence cost under $\Psi_{m}$-DIO operations is given by

$C_{c, \psi}^{(1), \epsilon}(\rho)=\log \left\lceil\min \left\{R_{\Delta}(\omega)+1 \mid \omega \in \mathbb{D}, F(\rho, \omega) \geqslant 1-\epsilon\right\}\right\rceil$.

Proof. Using a twirling argument similar to the distillation case, we can, without loss of generality, limit ourselves to operations of the form $\Lambda=\Lambda \circ \mathcal{T}$, which take the form

$$
\Lambda(Q)=\left\langle\Psi_{m}, Q\right\rangle X+\left\langle\mathbb{1}-\Psi_{m}, Q\right\rangle Z
$$

for some operators $X, Z$. The complete positivity and trace preservation of $\Lambda$ impose that $X, Z$ are valid quantum states. To impose that $\Lambda \in \Psi_{m}$-DIO, we have that

$$
\Delta(X)=\Delta \circ \Lambda\left(\Psi_{m}\right)=\Lambda \circ \Delta\left(\Psi_{m}\right)=\frac{1}{m} X+\frac{m-1}{m} Z .
$$

Noticing that $\Lambda\left(\Psi_{m}\right)=X$, this means that the set of states $\omega$ such that $\omega=\Lambda\left(\Psi_{m}\right)$ for some $\Psi_{m}$-DIO protocol $\Lambda$ is precisely the set of states for which there exists a state $\sigma$ such that $\frac{1}{m} \omega+\frac{m-1}{m} \sigma=\Delta(\omega)$. It is easy to see that this is only possible if $\Delta(\sigma)=\Delta(\omega)$. Defining the function

$$
g(\omega):=\min \left\{\lambda \mid \frac{\omega+\lambda \sigma}{1+\lambda} \in \mathcal{I}, \sigma \in \mathbb{D}, \Delta(\sigma)=\Delta(\omega)\right\},
$$


it is not difficult to show that, in fact, $g(\omega)=R_{\Delta}(\omega)$ [12]. We then have that the one-shot coherence cost under $\Psi_{m}$-DIO is

$$
\begin{aligned}
& C_{c, \Psi_{m}-\mathrm{DIO}}^{(1), \varepsilon)} \\
& \quad=\log \lceil\min \{m \in \mathbb{R} \mid \omega \in \mathbb{D}, F(\rho, \omega) \\
& \left.\left.\quad \geqslant 1-\varepsilon, R_{\Delta}(\omega) \leqslant m-1\right\}\right\rceil \\
& \quad=\log \left\lceil\min \left\{R_{\Delta}(\omega)+1 \mid \omega \in \mathbb{D}, F(\rho, \omega) \geqslant 1-\varepsilon\right\}\right\rceil,
\end{aligned}
$$

as required.

\section{Exact dilution}

Zero-error dilution can be characterized straightforwardly, as the required coherence cost is simply

$$
C_{c, \Psi_{m}-\mathrm{DIO}}^{(1), 0}(\rho)=\left\lceil\log \left[R_{\Delta}(\rho)+1\right]\right\rceil .
$$

Note that

$$
R_{\Delta}(\rho)=\left\|\Delta(\rho)^{-1 / 2} \rho \Delta(\rho)^{-1 / 2}\right\|_{\infty}-1,
$$

which makes this quantity easy to express and compute. In particular, noting that for every state we have $\rho \leqslant$ $\operatorname{rank}(\Delta(\rho)) \Delta(\rho)[56]$, this straightforwardly shows that the maximally coherent state $\Psi_{m}$ can be transformed into any other state with rank $\Delta(\rho) \leqslant m$, and it acts as a "golden unit" under the operations $\Psi_{m}$-DIO. For a pure state, we have exactly [12]

$$
R_{\Delta}(\psi)=\operatorname{rank} \Delta(\psi)-1,
$$

which further simplifies the characterization.

We note the additivity of $\log \left[R_{\Delta}(\rho)+1\right]$, immediately establishing that the asymptotic zero-error coherence cost under $\Psi_{m}$-DIO is given by

$$
C_{c, \Psi_{m}-\mathrm{DIO}}^{\infty, 0}(\rho)=\log \left[R_{\Delta}(\rho)+1\right] .
$$

\section{General transformations}

We can generalize the approach of coherence dilution to give a simple sufficient condition for transformations to general states. For any $\omega \in \mathbb{D}$, we have the following:

Proposition 5. If $R_{\Delta}(\omega)+1 \leqslant \frac{1}{\left\langle\Pi_{\rho}, \Delta(\rho)\right\rangle}$, then there exists a $\rho$-DIO map such that $\Lambda(\rho)=\omega$.

Proof. Recalling that $R_{\Delta}(\omega)=$ $\min \{\lambda \mid \omega \leqslant(\lambda+1) \Delta(\omega)\}$, it is easy to see that for any $\lambda \geqslant R_{\Delta}(\omega)+1$, there exists a state $\sigma$ such that $\omega+(\lambda-1) \sigma=\lambda \Delta(\omega)$. By assumption, there exists, in particular, $\sigma \in \mathbb{D}$, which satisfies

$$
\omega+\left(\frac{1}{\left\langle\Pi_{\rho}, \Delta(\rho)\right\rangle}-1\right) \sigma=\frac{1}{\left\langle\Pi_{\rho}, \Delta(\rho)\right\rangle} \Delta(\omega) .
$$

With this choice of $\sigma$, define the map

$$
\Lambda(X)=\left\langle\Pi_{\rho}, X\right\rangle \omega+\left\langle\mathbb{1}-\Pi_{\rho}, X\right\rangle \sigma .
$$

This map is clearly completely positive and trace preserving (CPTP), and we have

$$
\begin{aligned}
\Lambda(\rho) & =\omega, \\
\Lambda(\Delta(\rho)) & =\left\langle\Pi_{\rho}, \Delta(\rho)\right\rangle \omega+\left[1-\left\langle\Pi_{\rho}, \Delta(\rho)\right\rangle\right] \sigma \\
& =\left\langle\Pi_{\rho}, \Delta(\rho)\right\rangle\left\{\omega+\left[\frac{1}{\left\langle\Pi_{\rho}, \Delta(\rho)\right\rangle}-1\right] \sigma\right\} \\
& =\Delta(\omega),
\end{aligned}
$$

as required.

The above condition is, in general, not necessary for $\rho$ DIO transformations, and indeed, in general, $R_{\Delta}(\rho)+1>$ $\frac{1}{\left\langle\Pi_{\rho}, \Delta(\rho)\right\rangle}$, which means that even the trivial transformation $\rho \rightarrow \rho$ might not satisfy the condition of the proposition.

When the input state is a qubit, however, the transformations can be exactly characterized (see, also, [57,58]). We will, in particular, establish an equivalence between $\rho$-DIO and DIO in such transformations.

Proposition 6. For any single-qubit states $\rho$ and $\sigma$, the transformation $\rho \rightarrow \sigma$ is possible under $\rho$-DIO if and only if it is possible under DIO.

Proof. Clearly, if a DIO transformation $\rho \rightarrow \sigma$ exists, then so does a $\rho$-DIO transformation by the inclusion DIO $\subseteq \rho-$ DIO. By Theorem 30 in Ref. [12], the DIO transformation is possible if and only if $R_{\Delta}(\rho) \geqslant R_{\Delta}(\sigma)$ and $\|\rho\|_{\ell_{1}} \geqslant\|\sigma\|_{\ell_{1}}$. Since $R_{\Delta}$ is trivially a $\rho$-DIO monotone, as discussed earlier, it suffices to show that $\|\cdot\|_{\ell_{1}}$ is a $\rho$-DIO monotone, which will mean that the existence of a $\rho$-DIO transformation implies the existence of a DIO transformation.

To see that this is indeed true, note that $\|\rho-\Delta(\rho)\|_{1}$ is clearly a $\rho$-DIO monotone due to the contractivity of the trace distance under CPTP maps. But for a single-qubit state $\rho=\left(\begin{array}{cc}\rho_{00} & \rho_{01} \\ \rho_{01}^{*} & \rho_{11}\end{array}\right)$, we have $\|\rho-\Delta(\rho)\|_{1}=2\left|\rho_{01}\right|=\|\rho\|_{\ell_{1}}-$ 1 , which means that $\|\rho\|_{\ell_{1}}$ is also a $\rho$-DIO monotone for all single-qubit states.
[1] A. Streltsov, G. Adesso, and M. B. Plenio, Rev. Mod. Phys. 89, 041003 (2017).

[2] J. Aberg, arXiv:quant-ph/0612146.

[3] T. Baumgratz, M. Cramer, and M. B. Plenio, Phys. Rev. Lett. 113, 140401 (2014).

[4] A. Winter and D. Yang, Phys. Rev. Lett. 116, 120404 (2016).

[5] M. Horodecki and J. Oppenheim, Int. J. Mod. Phys. B 27, 1345019 (2012).

[6] E. Chitambar and G. Gour, Rev. Mod. Phys. 91, 025001 (2019).

[7] E. Chitambar and G. Gour, Phys. Rev. Lett. 117, 030401 (2016).
[8] I. Marvian and R. W. Spekkens, Phys. Rev. A 94, 052324 (2016).

[9] S. Du, Z. Bai, and X. Qi, Quantum Inf. Comput. 15, 1307 (2015).

[10] X. Yuan, H. Zhou, Z. Cao, and X. Ma, Phys. Rev. A 92, 022124 (2015).

[11] B. Yadin, J. Ma, D. Girolami, M. Gu, and V. Vedral, Phys. Rev. X 6, 041028 (2016).

[12] E. Chitambar and G. Gour, Phys. Rev. A 94, 052336 (2016).

[13] J. I. de Vicente and A. Streltsov, J. Phys. A: Math. Theor. 50, 045301 (2017). 
[14] Z.-W. Liu, X. Hu, and S. Lloyd, Phys. Rev. Lett. 118, 060502 (2017).

[15] H. Zhu, M. Hayashi, and L. Chen, J. Phys. A: Math. Theor. 50, 475303 (2017).

[16] A. Streltsov, S. Rana, P. Boes, and J. Eisert, Phys. Rev. Lett. 119, 140402 (2017).

[17] Q. Zhao, Y. Liu, X. Yuan, E. Chitambar, and X. Ma, Phys. Rev. Lett. 120, 070403 (2018).

[18] B. Regula, K. Fang, X. Wang, and G. Adesso, Phys. Rev. Lett. 121, 010401 (2018).

[19] E. Chitambar, Phys. Rev. A 97, 050301(R) (2018).

[20] D. Egloff, J. M. Matera, T. Theurer, and M. B. Plenio, Phys. Rev. X 8, 031005 (2018).

[21] K. Fang, X. Wang, L. Lami, B. Regula, and G. Adesso, Phys. Rev. Lett. 121, 070404 (2018).

[22] T. Theurer, D. Egloff, L. Zhang, and M. B. Plenio, Phys. Rev. Lett. 122, 190405 (2019).

[23] B. Regula, L. Lami, and A. Streltsov, Phys. Rev. A 98, 052329 (2018).

[24] Q. Zhao, Y. Liu, X. Yuan, E. Chitambar, and A. Winter, IEEE Trans. Inf. Theory 65, 6441 (2019).

[25] L. Lami, B. Regula, and G. Adesso, Phys. Rev. Lett. 122, 150402 (2019).

[26] L. Lami, arXiv:1902.02427, doi:10.1109/TIT.2019.2945798.

[27] S. Meznaric, S. R. Clark, and A. Datta, Phys. Rev. Lett. 110, 070502 (2013).

[28] T. Biswas, M. García Díaz, and A. Winter, Proc. R. Soc. London A 473, 20170170 (2017).

[29] M. A. Nielsen, Phys. Rev. Lett. 83, 436 (1999).

[30] S. Du, Z. Bai, and Y. Guo, Phys. Rev. A 91, 052120 (2015).

[31] H. Zhu, Z. Ma, Z. Cao, S.-M. Fei, and V. Vedral, Phys. Rev. A 96, 032316 (2017).

[32] K. Bu and C. Xiong, Quantum Inf. Comput. 13, 1206 (2017).

[33] K. Korzekwa, C. T. Chubb, and M. Tomamichel, Phys. Rev. Lett. 122, 110403 (2019).

[34] C. T. Chubb, M. Tomamichel, and K. Korzekwa, Phys. Rev. A 99, 032332 (2019).
[35] B. Morris and G. Adesso, J. Phys. A: Math. Theor. 51, 414007 (2018).

[36] F. Buscemi, Commun. Math. Phys. 310, 625 (2012).

[37] F. Buscemi and G. Gour, Phys. Rev. A 95, 012110 (2017).

[38] J. M. Renes, J. Math. Phys. 57, 122202 (2016).

[39] F. Buscemi and N. Datta, IEEE Trans. Inf. Theory 56, 1447 (2010).

[40] L. Wang and R. Renner, Phys. Rev. Lett. 108, 200501 (2012).

[41] M. Tomamichel and M. Hayashi, IEEE Trans. Inf. Theory 59, 7693 (2013).

[42] M. Hayashi, Quantum Information Theory: Mathematical Foundation (Springer, New York, 2016).

[43] M. Hayashi, arXiv:1709.07701.

[44] F. Dupuis, L. Krämer, P. Faist, J. M. Renes, and R. Renner, in XVIIth International Congress on Mathematical Physics (World Scientific, Singapore, 2012), pp. 134-153.

[45] T. Ogawa and H. Nagaoka, IEEE Trans. Inf. Theory 46, 2428 (2000).

[46] T. Ogawa and M. Hayashi, IEEE Trans. Inf. Theory 50, 1368 (2004).

[47] M. Horodecki, A. Sen(De), and U. Sen, Phys. Rev. A 67, 062314 (2003).

[48] D. Petz, Rep. Math. Phys. 23, 57 (1986).

[49] F. Buscemi, arXiv:1505.00535.

[50] F. Buscemi, Probl. Inf. Transm. 52, 201 (2016).

[51] R. Takagi and B. Regula, Phys. Rev. X 9, 031053 (2019).

[52] X. Wang and M. M. Wilde, Phys. Rev. Research 1, 033170 (2019).

[53] F. Buscemi, D. Sutter, and M. Tomamichel, Quantum 3, 209 (2019).

[54] R. Bhatia, Matrix Analysis (Springer, New York, 1996).

[55] G. Vidal, J. Mod. Opt. 47, 355 (2000).

[56] M. Hayashi, arXiv:quant-ph/0107004.

[57] P. M. Alberti and A. Uhlmann, Rep. Math. Phys. 18, 163 (1980).

[58] T. Heinosaari, M. A. Jivulescu, D. Reeb, and M. M. Wolf, J. Math. Phys. 53, 102208 (2012). 